\title{
Grußworte anlässlich des 50. Geburtstages von Prof. Helmut Flachberger
}

\author{
Wilfried Eichlseder \\ Leoben, Österreich
}

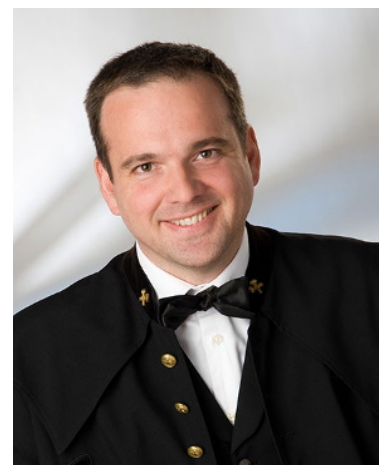

Univ.-Prof. Dipl.-Ing. Dr. mont. Helmut Flachberger

Am 17.11.2017 vollendet Univ.-Prof. Dipl.-Ing. Dr. mont. Helmut Flachberger ein halbes Jahrhundert an Lebensjahren. Geboren und aufgewachsen im Salzkammergut, studierte er an der Montanuniversität Bergwesen und dissertierte anschließend bei Univ.-Prof. Steiner am Institut für Aufbereitung. Im Rahmen seiner Dissertation beschäftigte er sich mit der Aufbereitung von Salzen und leistete damit einen wichtigen Beitrag zur Weiterentwicklung der Österreichischen Salinen. Was er sich damals vermutlich noch nicht gedacht, aber vielleicht gewünscht hatte, war, dass er eines Tages die Leitung dieses Lehrstuhls als Univ.-Prof. für Aufbereitung und Veredlung übernehmen sollte.

Im Jahre 2005 war es so weit: Prof. Flachberger wurde an die Montanuniversität berufen. Hier hat er in seinen wissenschaftlichen Arbeiten den Fachbereich der Trockenaufbereitung und dabei insbesondere das Gebiet der Elektroscheidung in wesentlichen Bereichen weiterentwickelt. Darüber hinaus arbeitet er auch auf dem Gebiet der elektrodynamischen Zerkleinerung und setzt damit die Zerkleinerungsforschung seines Vorgängers, Prof. Hans Jörg Steiner, mit neuen Ansätzen fort. Bedeutende Beiträge kamen von Prof. Flachberger auch zu Entwicklungen in der Aufbereitungsindustrie, wie dem neuen Bläh-Material „Bublon”, das er gemeinsam mit der Fa. Binder entwickelt hat. Nicht unerheblich ist auch der Umfang des wissenschaftlichen Nachwuchses: Unter der Betreuung von Helmut Flachberger sind bisher 20 Dissertanten hervorgegangen.

Wesentlich ist auch sein Einsatz für die Lehre. Neben den umfangreichen Lehrveranstaltungen leitet er seit vielen Jahren die Curriculumskommission Rohstoffingenieurwesen. Prof. Flachberger kümmert sich auch sehr intensiv um die Fortbildung der Aufbereiter und hat dafür einen Universitätslehrgang aus der Taufe gehoben. Darüber hinaus hat er über den BVÖ die jährliche Aufbereitertagung zu einem wesentlichen Seminarereignis mit jeweils mehreren hunderten Teilnehmern entwickelt und trägt damit zur Positionierung Leobens als Tagungsstandort bei.

Helmut Flachberger zeichnet sich durch eine sehr kooperative Art aus, was wesentlich dazu beigetragen hat, dass er zum Vorsitzenden des Professorenverbandes gewählt wurde und er diese Funktion nun seit 2 Jahren ausübt. Mit Umsicht und Effizienz leitet er die Sitzungen und vertritt die Belange der Professoren. Nicht unerwähnt soll auch die erfolgreiche jährliche Organisation der Sommerredoute der Montanuniversität bleiben, die sich unter seiner Leitung zu einem fixen Bestandteil im gesellschaftlichen Leben Leobens entwickelt hat.

Ich habe die Zusammenarbeit mit Prof. Flachberger immer sehr geschätzt, möchte mich dafür sehr herzlich bedanken und wünsche ihm noch viele interessante wissenschaftliche Herausforderungen und vor allem Gesundheit!

Glück auf!

Wilfried Eichlseder

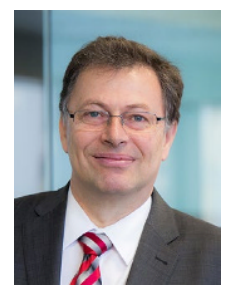

Univ.-Prof. Dipl.-Ing. Dr. techn. Dr. h.c. Wilfried Eichlseder, Rektor der Montanuniversität Leoben 